\title{
The coupled effects of mantle mixing and a water-dependent viscosity on the surface ocean
}

\author{
Kiran Chotalia ${ }^{\mathrm{a}, *}$, Neil Cagney ${ }^{\mathrm{b}}$, Carolina Lithgow-Bertelloni ${ }^{\mathrm{c}}$, John Brodholt ${ }^{\mathrm{a}, \mathrm{d}}$ \\ a Department of Earth Sciences, University College London, Gower Street, London, WC1E 6BT, UK \\ b School of Engineering and Materials Science, Faculty of Science and Engineering, Queen Mary University of London, Mile End Road, London, E1 4NS, UK \\ c Department of Earth, Planetary, and Space Sciences, University of California, Los Angeles, 595 Charles Young Drive East, Los Angeles, CA 90095-1567, USA \\ d Centre for Earth Evolution and Dynamics (CEED), University of Oslo, 0316 Oslo, Norway
}

\section{A R T I C L E I N F O}

\section{Article history:}

Received 6 December 2018

Received in revised form 27 July 2019

Accepted 2 October 2019

Available online $\mathrm{xxxx}$

Editor: B. Buffett

\section{Keywords:}

mixing

water-dependent viscosity

sea level

parametrised mantle convection

\begin{abstract}
A B S T R A C T
Water content plays a vital role in determining mantle rheology and thus mantle convection and plate tectonics. Most parameterised convection models predict that Earth initially underwent a period of rapid degassing and heating, followed by a slow and sustained period of regassing and cooling. However, these models assume water is instantaneously mixed and homogeneously distributed into the mantle. This is a limitation because the mixing time for water entering and leaving the mantle is a function of the Rayleigh number which varies dramatically with water content, temperature, and through time. Here we present an adapted parametrised model (Crowley et al., 2011) to include the coupled effects of the time scale of mixing with a water-dependent viscosity. We consider two mixing types: first, where the mixing time is constant throughout the model and second, where mixing time varies as a response to an evolving Rayleigh number. We find that, facilitated by the effects of water content in the melt region at mid-ocean ridges, a constant mixing time can induce long periods of degassing. The inclusion of a variable mixing time dependent on the Rayleigh number acts to limit the period of degassing and also results in more water being stored in the mantle and less at the surface than in both the constant and instantaneous mixing cases. Mixing time cannot be more than $\sim 2$ billion years as large mixing times trap water in the mantle, leaving a dry surface. Even small changes in the surface ocean induced by mixing times on the order of 0.1 Gyrs can cause changes in the global-mean sea level on the order of 10 's of metres. These changes in sea level could easily uncover topographic highs in the bathymetry, potentially aiding sub-aerial erosion a process thought to be important in early Earth evolution. Even in this relatively simple model, the inclusion of a mixing time between water entering and leaving the mantle creates a more dynamic water cycle.
\end{abstract}

(C) 2019 The Author(s). Published by Elsevier B.V. This is an open access article under the CC BY license (http://creativecommons.org/licenses/by/4.0/).

\section{Introduction}

Viscosity is the determinant physical property in the thermal evolution of the mantle, dependent on many parameters including temperature, pressure and grain size but the effect of water is currently undergoing investigation. Laboratory experiments on olivine have shown that water content can cause a reduction in viscosity of up to three orders of magnitude (e.g. Mei and Kohlstedt, 2000; Fei et al., 2013) and first principles simulations on perovskite show almost no effect (Muir and Brodholt, 2018). Understanding this relationship between mineral viscosity and water content is of particular interest due to the implications for mantle convec-

\footnotetext{
* Corresponding author.

E-mail address: kiran.chotalia.11@ucl.ac.uk (K. Chotalia).
}

tion and hence planetary evolution. If viscosity can vary several orders of magnitude, the convective vigour could also change by this magnitude with consequences for the style of convection.

The effects of a water-dependent viscosity on thermal evolution have been examined previously in parametrised (Crowley et al., 2011; Sandu et al., 2011) and two-dimensional (2D) models (e.g. Nakagawa et al., 2015). Parametrised studies have shown a water-dependent viscosity initially produces a period of heating and a state of net degassing (where degassing at mid-ocean ridges, MORs, exceeds regassing at subduction zones), followed by a long and sustained period of cooling and a state of net regassing (Crowley et al., 2011; Sandu et al., 2011). This is due to the rapidly decreasing water content increasing the viscosity, trapping heat and causing the temperature to build up. After some time, the increase in temperature lowers the viscosity and increases $R a$ such that the mantle starts to convect more efficiently again and the 


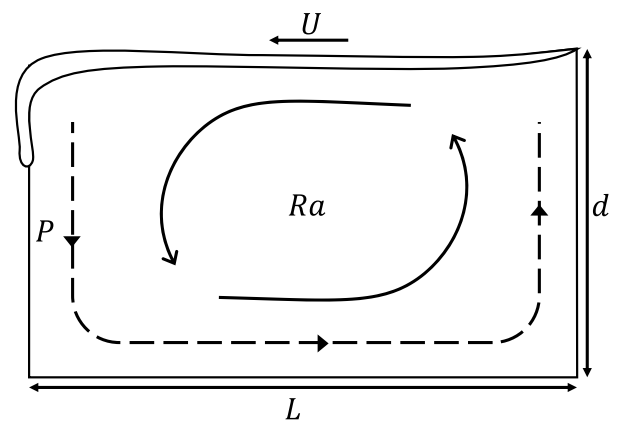

Fig. 1. The path, $P$, from subduction zone to mid-ocean ridge (MOR) represents the path of a subducting package. The time for the package to travel along this path is the mixing time, $\tau$. For constant mixing, $\tau$ is constant over the course of the model and the time to get from the subduction zone to the MOR is constant. For variable mixing, $\tau$ is proportional to the convective vigour, $\mathrm{Ra}^{2 / 3}$ and hence temperature and water content through viscosity. The travel time between subduction zone and MOR is dependent on the average mantle temperature $T$ and local water content at the ridge $X\left[\tau_{(R a)}\right]$, through $R a$.

mantle ends up in a period of gradual cooling and regassing. Some models suggest more than one cycle of heating and cooling have occurred over the lifetime of the Earth (Sandu et al., 2011). The initial period of degassing removes almost all of the water from the mantle reservoir resulting in a drier mantle, something that has been suggested to aid the onset of plate tectonics (Korenaga, 2011). A water-dependent viscosity has also been coupled with continent generation showing that increased continental weathering due to the presence of life on Earth favours a wet mantle (Höning et al., 2014; Höning and Spohn, 2016).

One of the biggest assumptions in parametrised models is that mixing of water introduced at subduction zones and extracted at MORs is instantaneous and moreover, water is homogeneously distributed throughout the mantle. This, of course, is a great simplification. It is clear that both water content and mixing time play vital roles in mantle evolution, and the two variables are strongly interdependent. In spite of this, there has been very little work attempting to understand this interdependence or what impact this time delay may have on the evolution of the Earth.

There is a rich literature on dynamical systems with a timedelay, which are found in many fields such as non-linear optics, population dynamics and physiology (May, 1980; Mackey and Glass, 1977; Niculescu et al., 1998). The introduction of a timedelay, such as the mixing time in the mantle, can cause the system to become unstable (Niculescu et al., 1998; Doyne Farmer, 1982). The role of the time-delay has been explored using model equations, such as the Mackley-Glass equation (Mackey and Glass, 1977), in which a progressive increase in the magnitude of the time-delay can cause the system to transition from a simple periodic response to a complex multi-component response and ultimately to chaos (Junges and Gallas, 2012). Such transitions can have a major impact on the final state of the system, and in terms of the mantle, the mixing time has the potential to significantly affect the mantle temperature, rates of mantle convection and sea level. Hence, we impose a mixing time in the water cycle of a thermal evolution model to understand the effects of a heterogeneous mantle water content introduced by subduction.

\section{Methods}

Consider an Earth-like planet with plate tectonics where a subducted package follows the trajectory shown in Fig. 1, travelling a total distance $P=2 d+L$. The time it takes to reach the MOR is the mixing time $\tau=P / U . \tau$ is also likely to depend on the convective vigour, $R a$ (where $R a$ is the ratio of buoyant to dissipative forces). As the velocity $U \propto R a^{\frac{2}{3}}$ (Turcotte and Oxburgh, 1967), $\tau \propto R a^{-\frac{2}{3}}$
Table 1

Starting conditions and viscosity constants for instantaneous mixing cases. For both viscosity laws, the mixing times are tested between 0.002 and 9 Gyrs on the orders of $0.001,0.01,0.1$ and 1 Gyrs.

\begin{tabular}{lcccl}
\hline & $\eta(T)$ & & $\eta(T, X)$ & Units \\
\hline$M_{s}\left(t_{0}\right)$ & 1.723 & & 1.887 & $1.39 \times 10^{21} \mathrm{~kg}$ \\
$T\left(t_{0}\right)$ & & 2500 & & $\mathrm{~K}$ \\
$H_{s f}$ & 1.706 & \multicolumn{2}{c}{1.724} & - \\
$\eta_{0}$ & & $7.53 \times 10^{15}$ & $\mathrm{~Pa} \mathrm{~s}$ \\
$r$ & 0 & & 0.3 & - \\
$R$ & & 8.314 & $\mathrm{~J} \mathrm{~mol} \mathrm{~K}^{-1}$ \\
$E$ & & $3 \times 10^{5}$ & $\mathrm{~J}$ \\
\hline
\end{tabular}

(Samuel et al., 2011) where mixing time is inversely proportional to convective vigour. This implies that in the past when the mantle was hotter, viscosity was low, $R a$ was high and the mixing time was short. As it cools, the viscosity increases; $R a$ decreases and $\tau$ becomes longer, assuming plate tectonics has operated since mantle solidification.

\subsection{Evolution model}

The evolution is solved by following the procedure outlined by Crowley et al. (2011). The convective vigour is described by the Rayleigh number

$R a=\frac{\alpha \rho g T d^{3}}{\kappa \eta}$

where $\alpha$ is thermal expansivity, $\rho$ is density, $g$ is gravity, $T$ is average mantle temperature, $d$ is mantle depth, $\kappa$ is thermal diffusivity and $\eta$ is viscosity. The conservation of energy

$\frac{d T}{d t}=\frac{-Q_{s}+H}{\rho V c_{p}}$

and conservation of mass

$\frac{d X}{d t}=\frac{R-D}{\rho V}$

equations are solved via fourth order Runge-Kutta (RK4) methods where $Q_{s}$ is surface heat flow, $H$ is heating from radiogenic elements, $V$ is mantle volume, $c_{p}$ specific heat capacity, average mantle water content is $X, R$ is regassing and $D$ is degassing. The conservation of energy depends on radiogenic heat production, $H$

$H=H_{s f} \sum_{j} \rho C_{j} H_{j} \exp \left(\frac{\ln 2\left(t_{p d}-t\right)}{\tau_{j}}\right)$

$H$ is a sum of the contributions from $\mathrm{U}^{238}, \mathrm{U}^{235}$, Th and $\mathrm{K}$ where $C_{j}$ is concentration (of the $j$ th element), $H_{j}$ is heat production, $\tau_{j}$ is radiogenic half life and $t_{p d}$ is present day time (Table 1). Eq. (2) also depends on the surface heat flow, $Q_{s}$

$Q_{s}=2 S k_{c} T\left(\frac{U}{\pi L \kappa}\right)^{\frac{1}{2}}$

where $U$ is plate velocity, $S$ is surface area, $k_{c}$ is thermal conductivity and $L$ is plate length. $Q_{s}$ is a function of $U$

$U=\frac{\kappa}{d}\left(\frac{L}{\pi d}\right)^{\frac{1}{3}} R a^{\frac{2}{3}}$

and is proportional to Ra. Degassing and regassing are also a function of $U$

$D=S F_{d} \frac{z_{m}}{L} U \rho X$ 


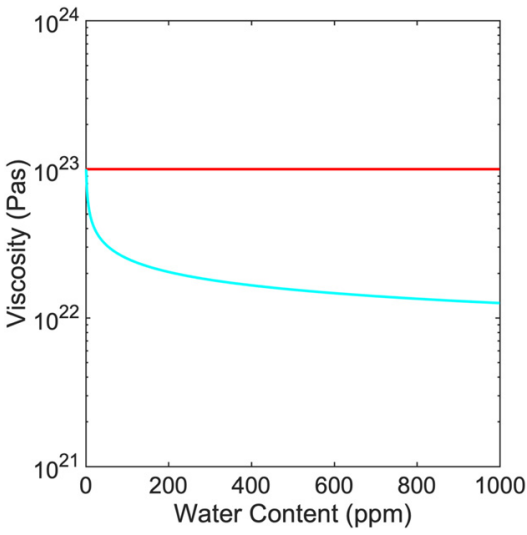

Fig. 2. Viscosity variation with respect to water content at $2200 \mathrm{~K}$ for both viscosity laws used in this model from Eq. (9). Both laws are calibrated to give the same viscosity when no water is present (Fei et al., 2013). The water-dependent law $\eta(T, X)$ varies one order magnitude for $1000 \mathrm{ppm}$.

$R=S F_{r} \frac{d_{l}}{L} U \rho X_{p}$

Degassing (Eq. (7)) defines the amount of water removed from the mantle at MORs. It also depends on the melting depth $z_{m}=$ $z_{1} T_{p}+z_{2} X+z_{3}$, a parametrised water-dependent melting depth (Hirschmann et al., 2009; Crowley et al., 2011) where $z_{1}, z_{2}$ and $z_{3}$ are constants and $T_{p}$ is potential temperature in degrees celsius (Mckenzie and Bickle, 1988) which, with water content, varies in time. The mass of melt is given by $z_{m} U \rho$ and it's water content is defined by the average mantle water content (when mixing is considered, this value will differ). Degassing is also dependent on the degassing efficiency, $F_{d}$. This constant defines how much water in the melting region escapes to the surface, where $1=100 \%$ of the water in the melt region reaches the surface reservoir.

Similarly, regassing (Eq. (8)) defines the amount of water subducted into the mantle. It is dependent on the evolving variables of thermal plate thickness $d_{l}$ and plate velocity. The thermal plate thickness is defined by the half space cooling model such that $d_{l}=2(\kappa L / U)^{\frac{1}{2}}$. Regassing efficiency $F_{r}$ describes the fraction of water that makes it past the subduction factory and $X_{p}$ is an average plate water concentration. The values of these constants and others defined here are found in Table A.2.

\subsection{Water-dependent viscosity}

We test two simplified viscosity laws of the form

$\eta=\eta_{0}\left(\frac{X}{X_{C}}\right)^{-r} \exp \left(\frac{E}{R T}\right)$

$\eta$ is the viscosity, $\eta_{0}$ is a calibration constant, $E$ is the activation energy and $R$ is the ideal gas constant. $r$ is the water content exponent, controlling whether the law is water-independent $(\eta(T)$ where $r=0)$ or water-dependent $(\eta(T, X)$ where $r=0.3)$ with an order of magnitude sensitivity to $1000 \mathrm{ppm}$ of water (Fei et al., 2013), shown in Fig. 2. $\eta_{0}$ is chosen such that for an average midmantle temperature of $2200 \mathrm{~K}$ and $\eta(T)$, viscosity is equivalent to a dry mid-mantle bridgmanite of $10^{23}$ Pa s (Ammann et al., 2010).

\subsection{Mixing time}

The mixing time, $\tau$, is incorporated into the evolution model in two ways: (1) $\tau$ is constant or (2) $\tau$ is variable. Fig. 3 illustrates where these steps occur with respect to solving Eqs. (2) and (3). The incorporation of a mixing time inherently requires the tracking of two different mantle water contents: the whole mantle average $X$ and a localised water content at the MOR $X\left[\tau_{(R a)}\right]$.

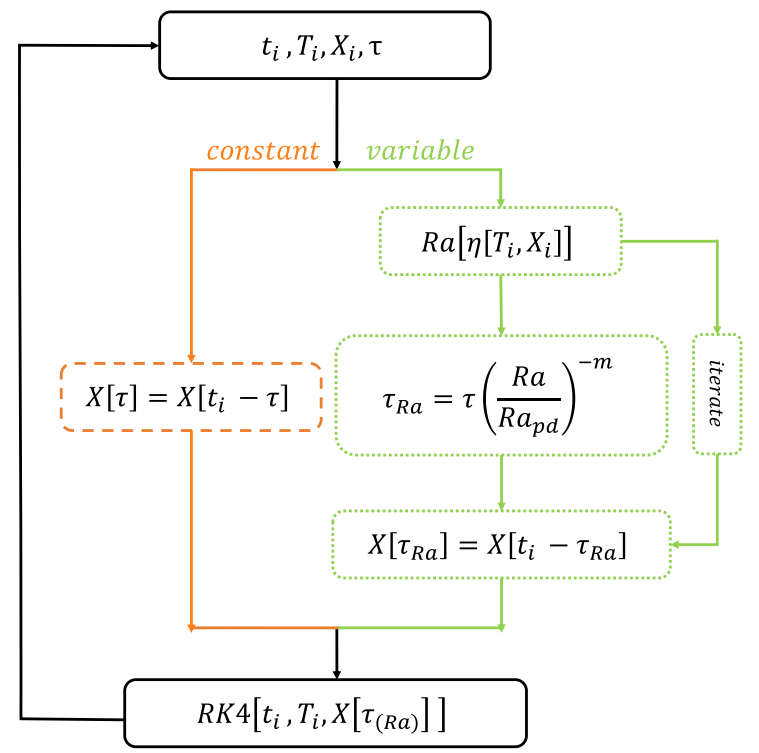

Fig. 3. Schematic representing the main calculation loop. Solid boxes represent the steps taken in all cases, the orange path with a dashed box represents the additional steps when mixing time is constant and the green path with dotted boxes represents the steps when mixing time is variable. $\tau$ is the constant mixing time or the prescribed mixing time that is later scaled by $R a$ to give $\tau_{R a}$ for variable mixing. Eqs. (2) and (3) are solved with a fourth order Runge-Kutta solver, RK4 with $X\left[\tau_{(R a)}\right]$ and hence the evolution is dependent on $X\left[\tau_{(R a)}\right]$ i.e. the local water content at the ridge. In contrast, $X$ is the global, average mantle water content, solved for over the course of the model. (For interpretation of the colours in the figure(s), the reader is referred to the web version of this article.)

This difference arises as it takes a finite amount of time for any subducted water to reach a MOR; this transit time across the conceptual model domain (Fig. 1) is the mixing time.

When mixing is instantaneous i.e. $\tau=0$, any water subducted into the mantle is homogeneously mixed into the mantle and can instantly be degassed into the surface ocean. This is represented in Fig. 3 by the arrows and lines outlined in black. Only $X$ and $T$ are used to calculate and solve Eqs. (1)-(9). For a constant mixing time, $\tau$, there is an imposed delay to when water reaches the ridge, shown by the orange path in Fig. 3. This water content is calculated as $X[\tau]=X\left[t_{i}-\tau\right]$. For example, if the mantle starts as dry, melting at the MOR is determined by dry conditions, before any water arrives at the ridge. Now, $X[\tau]$ is used to calculate and solve Eqs. (1)-(9). Eq. (3) still solves for the average mantle water content, however it is now dependent on the local conditions at the MOR, controlled by $X[\tau]$. The variable mixing time, $\tau_{R a}$, is calculated as

$\tau_{R a}=\tau\left(\frac{R a\left[T_{i}, X_{i}\right]}{R a_{p d}}\right)^{-m}$

where $R a$ is the Rayleigh number calculated with $X_{i}$ and $T_{i}$, $R a_{p d}=10^{6}$ and $m=2 / 3$ is the classical scaling between the plate velocity and Rayleigh number (Eq. (6)) for any given $\tau$. $\tau_{R a}$ evolves over the course of the model such that when $R a$ is high, convection is vigorous and $\tau_{R a}$ is short (and vice versa). $X\left[\tau_{R a}\right]=$ $X\left[t_{i}-\tau_{R a}\right]$ and Eqs. (1)-(9) are calculated therefore solved with a varying mixing time. In order to prevent the appearance of numerical instabilities, the calculation of $X\left[\tau_{R a}\right]$ involves iteration outlined in Fig. 3. $X\left[\tau_{R a}\right]$ is also taken to be the average $X$ found over five time steps, centred about $\tau_{R a}$. 


\subsection{Model set-up}

Instantaneous mixing cases $(\tau=0)$ are calibrated by tuning the initial ocean mass to give one ocean mass $\left(1.39 \times 10^{21} \mathrm{~kg}\right)$ at the present day (4.6 Gyrs) whilst for constant and variable mixing, mantle water content is allowed to evolve freely to assess the effects of mixing on the water cycle. By tuning $H_{s f}$, the surface heat flow for all models is constrained to be within the range 45-46 TW at the present day (Lay and Buffett, 2008).

Where the water should reside at the beginning of the model is unclear. This model only considers convection in a solid mantle and hence assumptions must be made about what happens to water prior to or during the freezing of a magma ocean, and also after the Moon-forming impact. Water is contributed primarily during planetary accretion (Marty et al., 2016), although as the planet continues to form, it is unclear where water resides. Impact events can form and degas magma oceans and liquid water on the Earth's surface during times of giant impacts may be likely (Tucker and Mukhopadhyay, 2014). Models of a solidifying magma ocean have shown that the most effective degassing phase of volatiles is during magma ocean solidification (Elkins-Tanton, 2011) but conversely the sinking of hydrous cumulates enriches the upper mantle in water, suggesting that the vast majority of accretionary water can be held in the mantle interior (Tikoo and Elkins-Tanton, 2017). However, these models use mantle water capacity for wadsleyite and ringwoodite at present day mantle temperatures. Water capacity has been show to decrease with increasing temperatures (Ohtani et al., 2000) and hence today's most hydrous mantle minerals such as wadsleyite and ringwoodite are likely to hold much less than 3000 ppm (Ohtani et al., 2000) of water in the past. If the magma ocean is hydrous, as it solidifies the water is expelled to the surface.

Thus far, previous deep water cycle models have also shown that beginning with water in the mantle results in a short period of net degassing (Crowley et al., 2011; Sandu et al., 2011) in which almost all of the water is expelled to the surface. Models with further geochemical, petrological and geological constraints for Earth advocate for a drier mantle in the Hadean in order to take into account the initiation of plate tectonics (Korenaga, 2011). For these reasons, we assume a dry mantle and a surface reservoir holding approximately two ocean masses of water. Models testing a wet starting condition are discussed in Figs. A1 and A2.

We examine a suite of mixing times varying from 0.002 to 9 Gyrs, for constant and variable mixing and each viscosity law (Table 1). The case where the mixing time is 9 Gyrs is equivalent to only regassing operating as no water ever reaches a MOR to be degassed i.e. $X\left[\tau_{(R a)}\right]$ is always zero. Running models to 9 Gyrs ensures that we capture behaviour of the system until it reaches a steady state.

\section{Results}

Both the thermal evolution and water cycle can be in two states, depending on whether the input or output of the system is dominant. For thermal evolution, the planet can either be in a state of cooling where $Q s>H$ or heating where $Q s<H$. Similarly, the water cycle can be in a state of net regassing where $R>D$ or net degassing where $R<D$. In general, the planet is cooling and regassing as shown previously (Crowley et al., 2011) and in Fig. A3. However, including mixing shows deviation from this trend.

\subsection{Water-independent viscosity}

\subsubsection{Instantaneous mixing}

Fig. 4 shows selected cases that represent the overall effects of a constant and variable mixing time for 9 Gyrs. The simplest case comprises of $\eta(T)$ with $\tau=0$, i.e. mixing is instantaneous. The mantle heats then cools to $\sim 2000 \mathrm{~K}$ (Fig. 4a) and increases in water content (Fig. 4b) to $\sim 425 \mathrm{ppm}$. As viscosity (Fig. 4c) is only dependent on temperature, the increase and decrease in temperature cause a decrease and increase in viscosity, respectively. The melting depth (Fig. 4e) is dependent on both temperature and water content. As a result, during the first 5 Gyrs the change in temperature is more significant than the change in water content and the melting depth decreases with decreasing temperature. After 5 Gyrs, the change in water content is more notable than the change in temperature and melting depth increases with increasing water content. This evolution agrees with previous parametrised studies (e.g. Korenaga, 2011).

\subsubsection{Constant mixing}

The addition of a constant mixing time has a pronounced effect in the water cycle, shown in Fig. 4 for a sample case of $\tau=1$ Gyrs. The water cycle has no effect on temperature evolution as $\eta(T)$, hence the differences are best illustrated in Figs. 4b, 4d and 4e.

During the first billion years, $X[\tau]$ is zero as no subducted water has reached a MOR (i.e. $t<\tau$ ). As there is no water feeding the MOR, no water is degassed during this period and the water cycle is in a state of net regassing.

Once $t>\tau$, local water content $X[\tau]$ becomes non-zero as water present in the mantle is sampled by MORs. The increase in local water content and the corresponding increase in melting depth allows degassing to begin. Degassing outpaces regassing and a period of net degassing is induced by $\sim 2$ Gyrs (Fig. $4 \mathrm{~b}$ ). For the remainder of the model, as $X[\tau]$ is lower than $X$, less water is removed than when mixing is instantaneous. This allows $X$ to increase to above the instantaneous case by 9 Gyrs. Net degassing causes a decrease in average mantle water content, and after 1 Gyrs in $X[\tau]$ as well. Decreasing local water content and melting depth reduces degassing such that net regassing resumes by $\sim 3$ Gyrs.

\subsubsection{Variable mixing}

In general, the introduction of a variable mixing time using ( $m=2 / 3$ ) causes various trends of the constant mixing case to resemble those observed for instantaneous mixing. This is the result of the dependency of mixing time on viscosity through $R a$. At the beginning of the model, high temperature and low viscosity cause short mixing times, but as the mantle cools and viscosity increases, the mixing time becomes longer (Fig. 4f). The period where local water content at the MOR is zero is shorter when the mixing time is variable.

This is illustrated in Fig. $4 \mathrm{f}$ by the line representing $t=\tau_{R a}$. The variable mixing case spends less time above the line (where $\left.X\left[\tau_{R a}\right]=0\right)$ than the constant mixing case. As the mixing time increases, less water reaches MORs and therefore less water can be degassed. This results in the absence of a period of net degassing and more water residing in the mantle for variable mixing than instantaneous mixing.

\subsubsection{Influence of mixing time scaling}

The data presented in Fig. 4 was calculated using Eq. (10) where $m=2 / 3$. To test the effect this scaling has on mantle evolution, we vary $m$ from 0 to 1 , where $m=0$ is equivalent to a constant mixing time. The results are shown in Fig. 5. As with Fig. 4, the temperature (Fig. 5a) and viscosity (Fig. 5c) are the same for each case. As $m$ is increased, $\tau_{R a}(t=0)$ decreases (Fig. $\left.5 f\right)$, and for $m=1$, the unscaled mixing time, $\tau$, of 1 Gyrs is reduced by an order of magnitude to 0.2 Gyrs. This decreases the period where $X\left[\tau_{R a}\right]=0$ in which the mantle goes through fluctuations in water content and an early period of degassing. This ceases when mixing time is strongly dependent on $R a(m \gtrsim 0.8)$, where water content increases smoothly and no period of degassing occurs. 
(a)

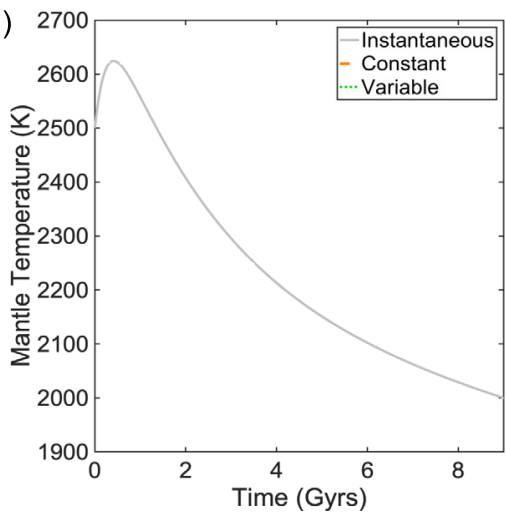

(d)

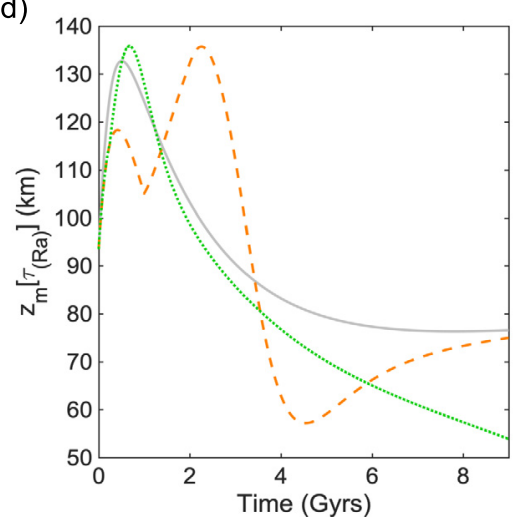

(b)

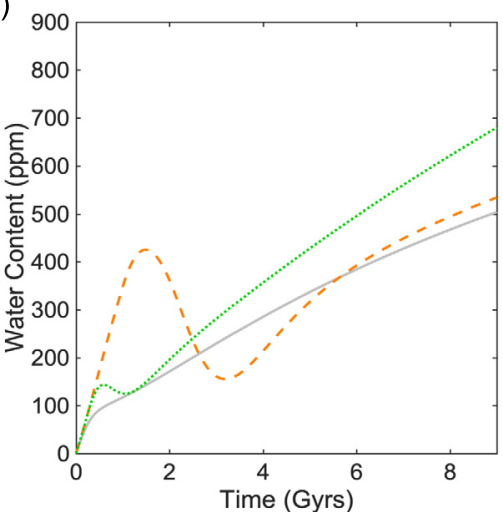

(e)

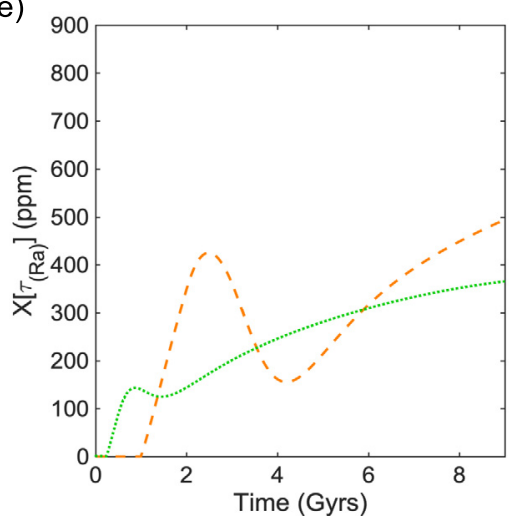

(c)

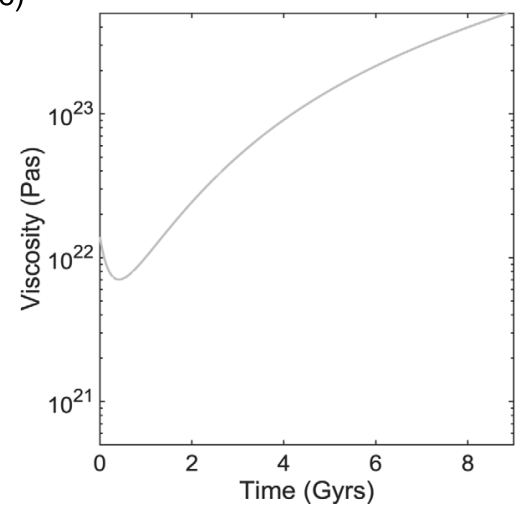

(f)

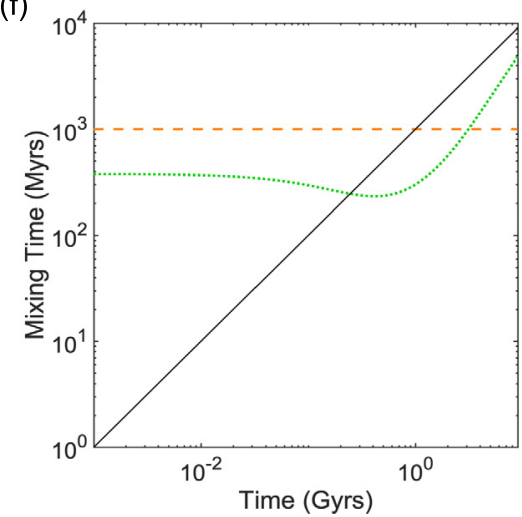

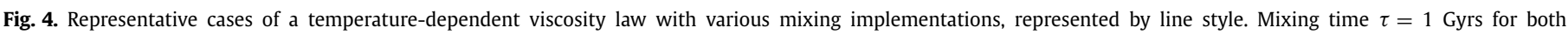

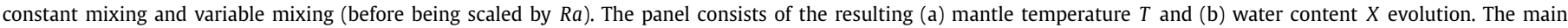

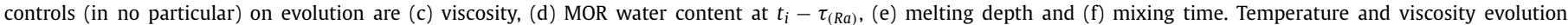

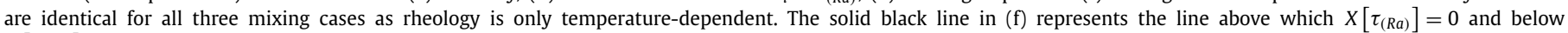

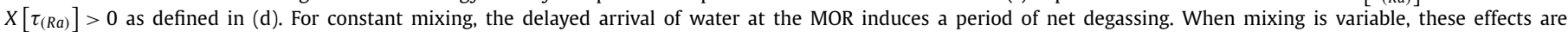
damped and net degassing begins earlier and lasts for a shorter amount of time.

For cases in which $m$ is small ( $\tau_{R a}$ is weakly dependent on $R a$ ), the initial period of net regassing is longer, leading to a greater build up of water in the mantle early on: for $m=0, X$ reaches a local maximum of $425 \mathrm{ppm}$ at $1.5 \mathrm{Ga}$. This excess of water eventually leads to a period of net degassing, lasting from $1.5 \mathrm{Ga}$ to 3.2 Ga. For cases with a slightly larger $m$ (mixing time is more sensitive to $R a$ ), the period of initial net regassing and the period of net degassing as the mantle readjusts become shorter, until by $m=0.8$ there is no maxima in $X$ and no net degassing. The duration of net degassing will be discussed further in Section 4.1 .

These cases may thus be divided into two sets: cases where mixing time is weakly dependent on $R a(m<0.8)$ with a period of net degassing and cases where mixing time is strongly dependent on $R a(m \geq 0.8)$ where there is no net degassing.

\subsection{Water-dependent viscosity}

\subsubsection{Instantaneous mixing}

Fig. 6 shows representative cases with a viscosity law that also depends on water content (Eq. (9)). The simplest case for $\eta(T, X)$ is also when mixing is instantaneous, i.e. $\tau=0$. Over 9 Gyrs, the mantle cools to $\sim 1800 \mathrm{~K}$ (Fig. $4 \mathrm{a}$ ) and water content increases to $\sim 600 \mathrm{ppm}$. The thermal evolution exhibits a short period of initial heating for $\sim 0.15$ Gyrs. During this time, water is being subducted into the mantle (Fig. 6b) and the increase in both temperature and water content contribute to a decrease in viscosity (Fig. 6c) and increase in melting depth (Fig. 6d). The decrease in viscosity allows surface heat flow to increase until it becomes greater than radiogenic heating and the mantle cools. The increase in melting depth and water content during the period of heating increases de- gassing. However, regassing remains dominant and the water cycle is in a state of net regassing for the entire model.

\subsubsection{Constant mixing}

Implementation of a constant mixing time has a similar effect as when $\eta(T)$. Some of the extreme variations in the mixing depth and other properties that were seen in the water-independent case have been damped; for constant mixing, the peak water content in the early Earth $(t<4 \mathrm{Ga})$ is $443 \mathrm{ppm}$. The oscillations in water content are associated with changes in the viscosity (Fig. 6c) and therefore also affect mantle temperature (Fig. 6a), which both exhibit fluctuations for $t<4 \mathrm{Ga}$.

During the first billion years when $X[\tau]=0$, heating is more pronounced than in the instantaneous mixing case. The water subducted during this time has not reached a MOR and therefore, surface heat flow is only dependent on temperature as before. The mantle feeding MORs is dry, local viscosity is relatively high and surface heat flow is lower than radiogenic heating. The mantle heats faster than the instantaneous case causing viscosity to decrease. This allows surface heat flow to increase until it becomes greater than radiogenic heating and the mantle begins to cool.

After 1 Gyrs, $X[\tau]>0$ and water subducted previously reaches the MOR. The increase in $X[\tau]$ decreases local viscosity at the MOR, increasing surface heat flow and causing a period of rapid cooling. As temperature rapidly decreases and local MOR water content increases (Fig. 6e), a local maximum in the melting depth forms (Fig. 6d), and degassing increases.

Around 2.5 Gyrs, $X[\tau]$ decreases with decreasing temperature, rapidly decreasing the melting depth. This reduces degassing and regassing dominates for the remainder of the model. 
(a)

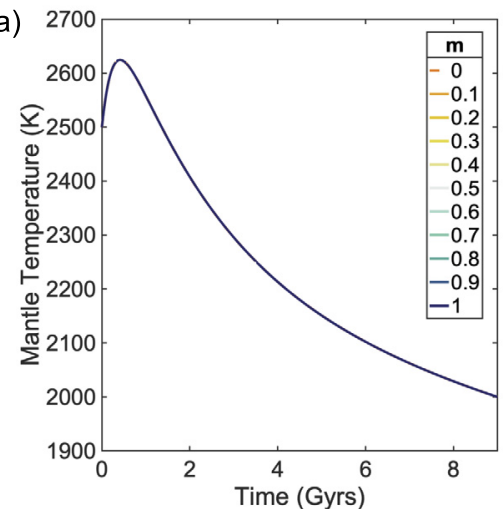

(d)

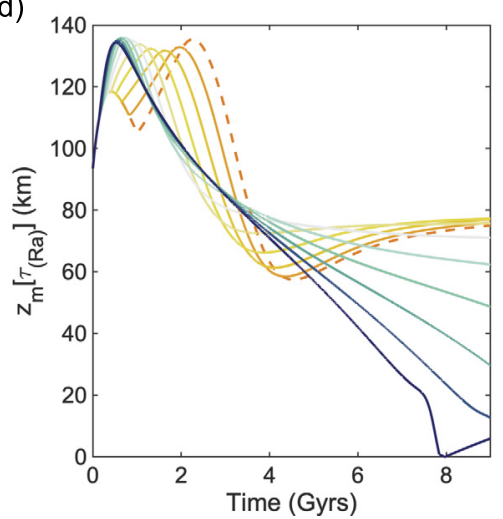

(b)

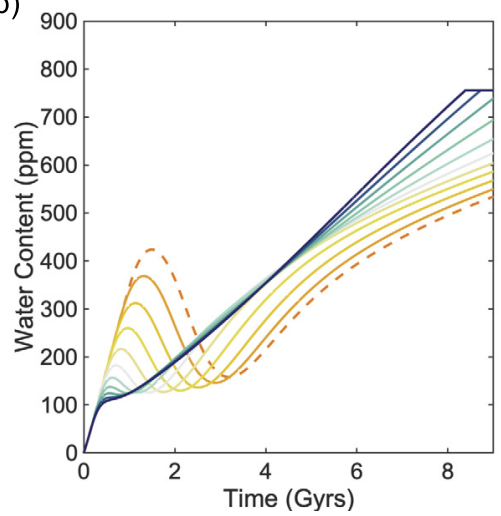

(e)

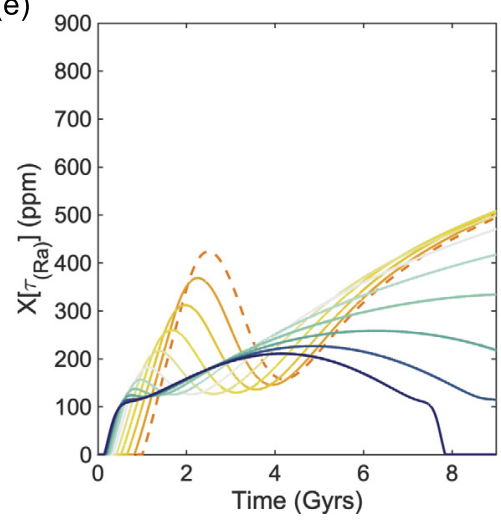

(c)

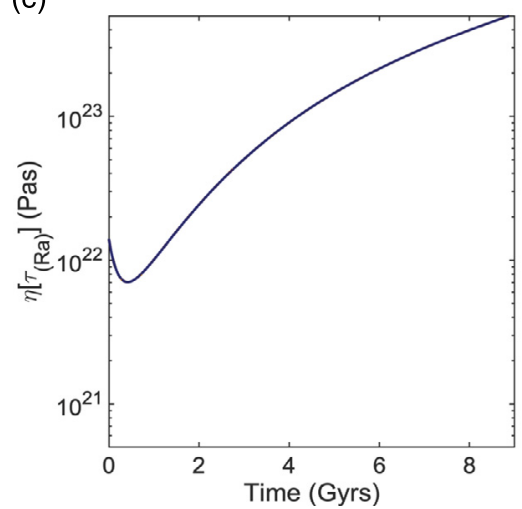

(f)

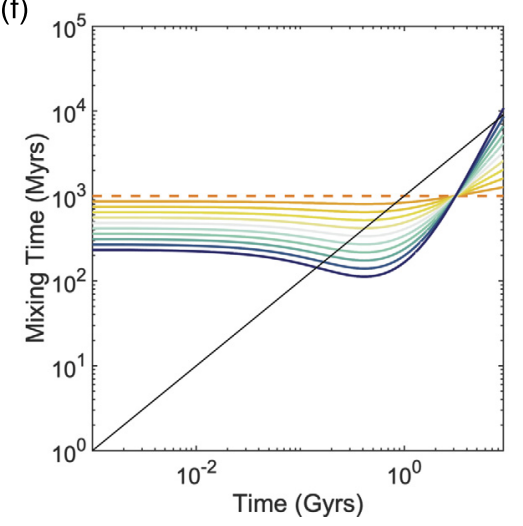

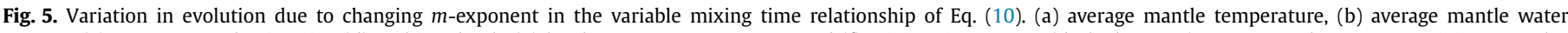

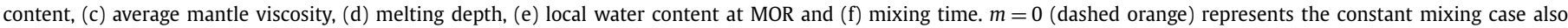

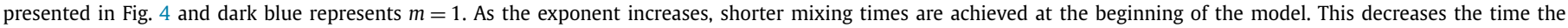
model spends with only regassing operating, eventually removing the period of net degassing and increasing the water content of mantle.

\subsubsection{Variable mixing}

The final layer of complexity comes with including a variable mixing time. As with $\eta(T)$, variable mixing closely resembles instantaneous mixing where a shorter mixing time at the beginning of the model causes a shorter period in which $X\left[\tau_{R a}\right]=0$ (Fig. 6e and $6 \mathrm{f}$ ). Less water is subducted and net degassing is no longer induced. However, unlike the water-independent case, mantle water content (Fig. 6b) exhibits stability for 0.2 Gyrs, which suggests that varying $\tau$ before it is scaled by $R a$ may cause net degassing to reappear for $\tau \neq 1 \mathrm{Gyr}$ (Eq. (10)). Differences in local viscosity and temperature evolution are also more comparable to those when mixing is instantaneous as the effects of degassing are dampened by variable mixing.

\subsubsection{Influence of mixing time scaling}

Varying $m$ when $\eta(T, X)$ has similar effects as when examining the differences seen in Figs. 4 and 6; the initial scaled mixing time (Fig. 7f) has a range of one order of magnitude with $m=1$ resulting in 0.1 Gyrs. This dampening effect of $\eta(T, X)$ results in less extreme behaviour. As with Fig. 6, there is also a feedback to the temperature (Fig. 7a) and viscosity (Fig. 7c) evolution. As with the water-independent case (Fig. 5), a period of net degassing is induced for $m<0.8$, showing that if the mixing time does not vary by orders of magnitude, net degassing is expected.

In summary, for a mixing time of 1 Gyrs, constant mixing $(m=0)$ induces a period of net degassing whilst variable mixing dampens that effect and resembles the instantaneous case (for $m>0.8$ ). A constant mixing time assumes subducted water takes 1 Gyrs to reach a MOR. This leads to an early build up of water in the mantle and a period of degassing as the mantle readjusts. In contrast, for cases where the mixing time is strongly dependent on
$R a$ (i.e. for $m \gtrsim 0.8$ ), the greater mantle temperatures in the early Earth ensure $\tau_{R a}$ is very small for much of Earth's history and as a result the mantle evolution closely resembles that seen for the instantaneous mixing case.

As the model progresses $(t \gtrsim 4 \mathrm{Ga})$, the trends in temperature (Fig. 7a) converge whilst trends in water content deviate. This is due to the deviations in $X\left[\tau_{R a}\right]$, as result of the variable mixing time (Fig. 7f). This indicates that even a weak dependence of mixing time on mantle conditions (i.e. $R a$ ) is sufficient to cause the mantle temperature evolution in the present day to resemble the idealised case of instantaneous mixing.

The mantle evolution is most sensitive to the mixing time in the early Earth; this is unsurprising, given this is the period in which changes in $R a$ are most rapid. The mantle evolution appears to be very sensitive to the mixing time at $t=0$, as this can lead to a build up of large quantities of water in the mantle followed by a period of degassing, highlighting the importance of initial conditions in the mantle for the evolution of the planet.

\section{Discussion}

\subsection{Periods of net degassing}

In Figs. 4-7, we present only the cases of mixing where $\tau=1$ Gyrs. However, estimates for the present day mixing time for Earth vary from a few hundred million years to a few billion years (Kellogg and Stewart, 1991; Samuel et al., 2011). This will inevitably have an impact on the transitions between regassing and degassing in the water cycle presented in the previous section.

When a mixing time is present, the water cycle can go through three phases: (1) initial net regassing when $X\left[\tau_{(R a)}\right]=0$, (2) net 
(a)

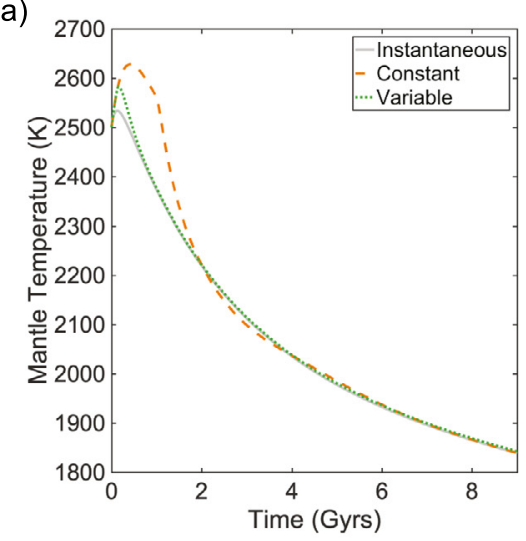

(d)

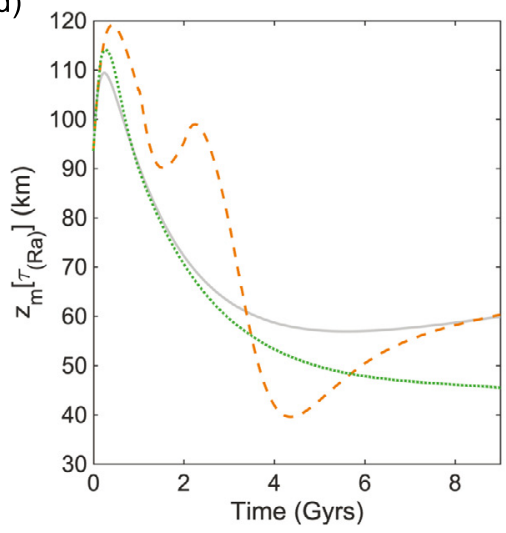

(b)

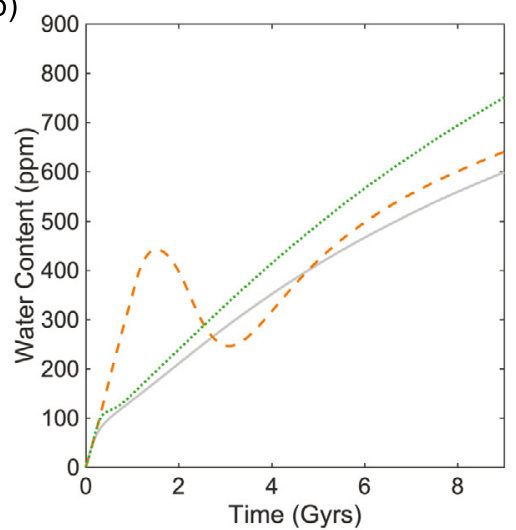

(e)

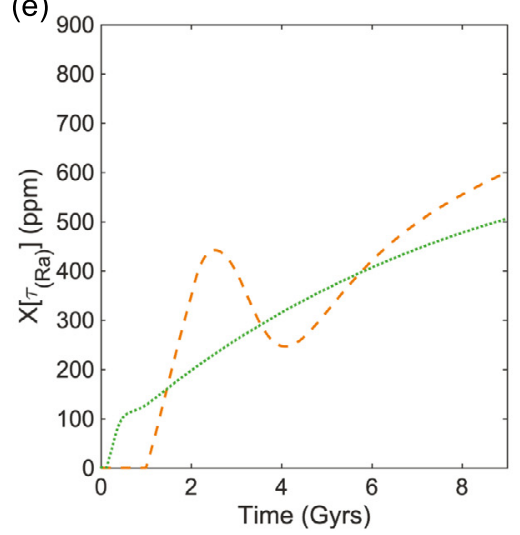

(c)

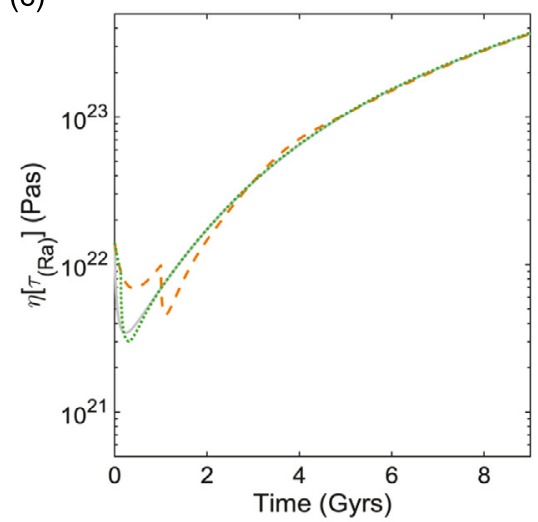

(f)

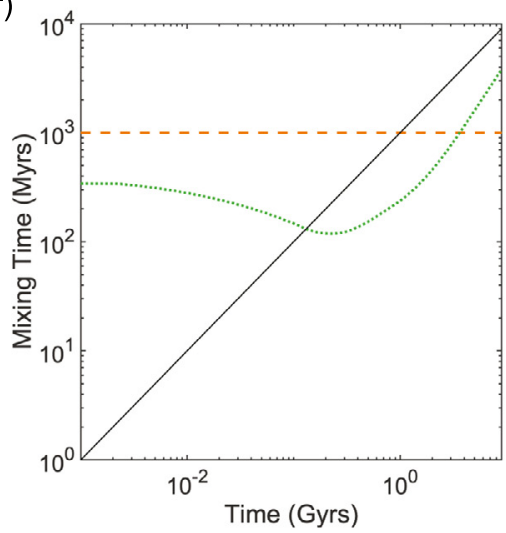

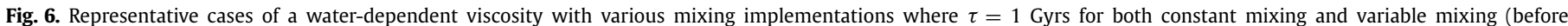

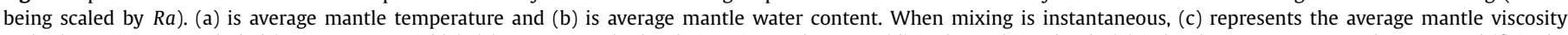

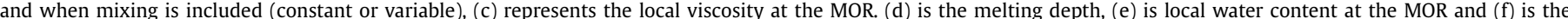

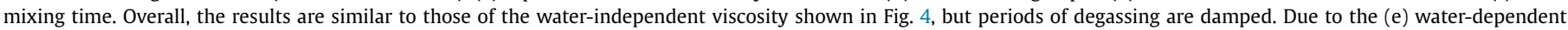
viscosity, there is also feedback to the temperature evolution through the surface heat flow.

degassing when $X\left[\tau_{(R a)}\right]$ initially becomes non-zero and (3) net regassing for the remainder of the model. These timings can be examined by extracting the turning points in $X$. These points represent where $\frac{d X}{d t} \sim 0$, i.e. when net degassing begins or ends. Fig. 8 presents data from the four different suites of models (constant and variable mixing for both water-independent and waterdependent viscosity laws) considered in the previous section, but here $\tau$ varies between 0.002 and 9 Gyrs. Cases with variable mixing times were performed using $m=2 / 3$. Fig. 8a shows the constant mixing time case for a water-independent viscosity where there are two turning points; the first indicates the start of net degassing and the second indicates the end of net degassing. For $\tau<0.2$ Gyrs, there are no turning points and the water cycle is always in a state of net regassing, behaving almost identically to the instantaneous mixing case. For $\tau>0.2$ Gyrs we see the beginning and end of a net degassing period. In general, as the mixing time increases, both the start and end of net degassing are delayed and the duration of net degassing increases. The first turning points follow a linear trend as the onset of water's influence on degassing is controlled by the end of the period where $X[\tau]$ is zero. The end points follow a linear trend until after $\tau=2.5$ Gyrs where end times begin to deviate.

Fig. 8b shows two suites of data for constant mixing and a water-dependent viscosity. The net degassing region looks similar to the water-independent viscosity case (Fig. 8a) with the period starting later and ending earlier. Unlike $\eta(T)$, the surface heat flow has to be scaled in order for the 46 TW condition to be met (Table A3) further shortening the duration of net degassing.
The addition of a variable mixing time has a drastic impact on net degassing. For a water-independent viscosity shown in Fig. 8c, a period of net degassing only occurs for $\tau=0.8-2$ Gyrs lasting $\sim 0.3-1.4$ Gyrs, respectively. In contrast, for the same period in constant mixing, net degassing lasts $\sim 1.4-3.5$ Gyrs, respectively. The period also occurs much earlier for variable mixing starting $\sim 0.6-1$ Gyrs when $\tau=0.8-2$ Gyrs compared with $\sim 1.2-3$ Gyrs for constant mixing, respectively. Hence, a variable mixing time causes net degassing to occur earlier and for a much shorter period, i.e. the start occurs later and the end occurs earlier, and only for a limited range of $\tau$.

When viscosity is dependent on water and the mixing time is variable, no net degassing occurs. This is due to the relatively short $\tau_{R a}$, calculated (Eq. (10)) as a result of increasing mantle temperature and increasing mantle water content, both decreasing $R a$. The effect of water content on viscosity (Eq. (9)) and hence $R a$ reduces $\tau_{R a}$ pushing evolution to behave more like the instantaneous mixing case where $\tau=0$. It is more likely to observe short periods of stability or slower changes in water content. For example, cases where $\tau=1-2$ Gyrs, the water content evolution behaves as in Fig. $6 \mathrm{~b}$ where mixing is variable. These results demonstrate how the existence and duration of a period of degassing is very sensitive to both the mantle viscosity and the estimates of the mantle mixing time.

When exploring the influence of mixing time, the inverse relationship is seen. For example, Fig. 9a shows periods of net degassing as a function of $\mathrm{m}$ in Eq. (10) for $\eta(T, X)$ (Fig. 7). Increasing $m$ decreases the time period when $t<\tau_{R a}$, therefore less water is solely regassed and the later influence on melting depth is not 
(a)

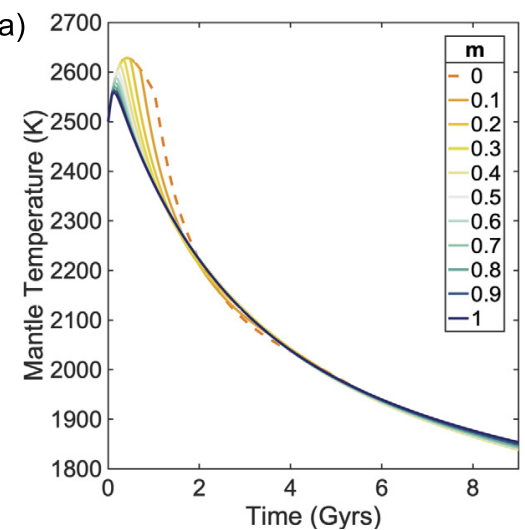

(d)

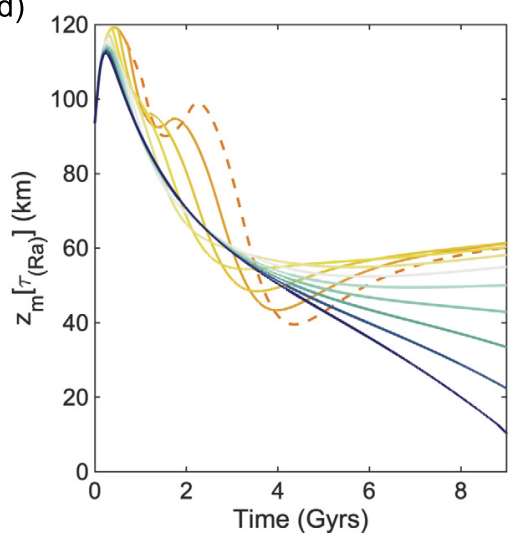

(b)

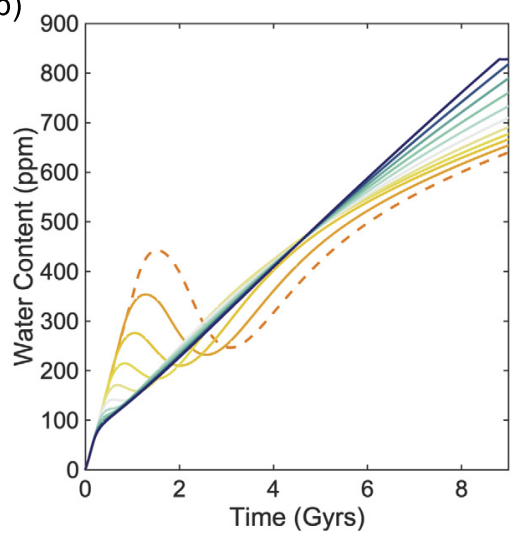

(e)

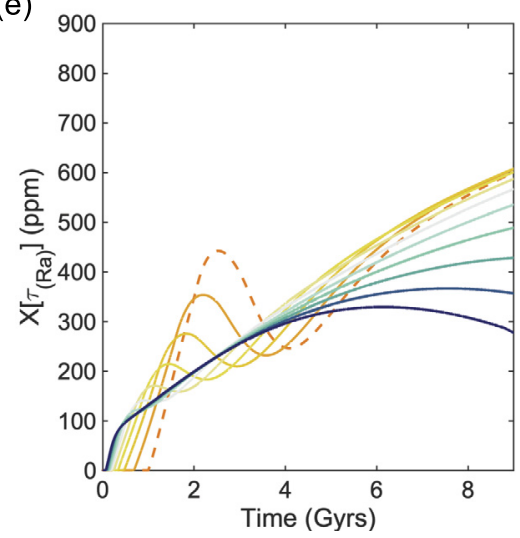

(c)

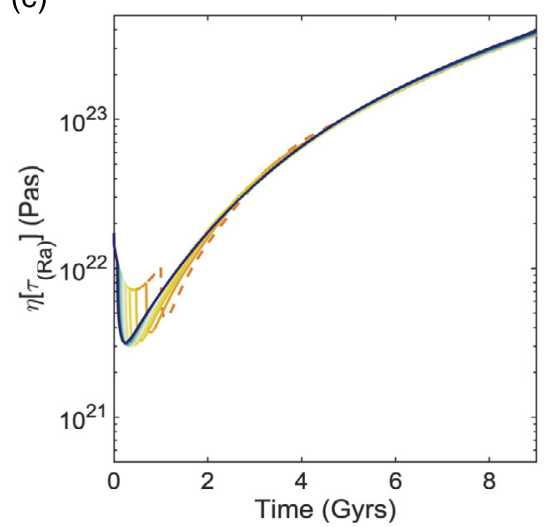

(f)

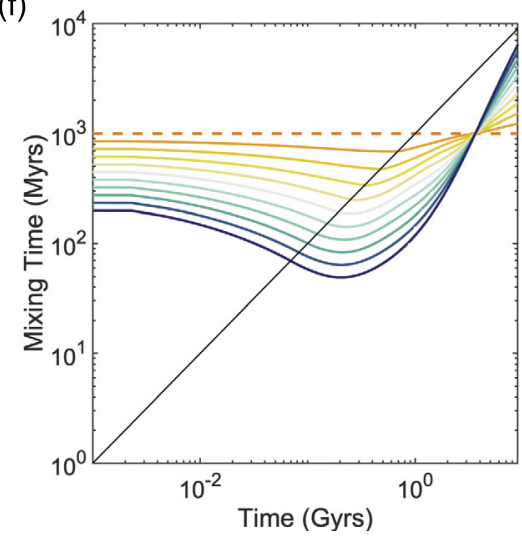

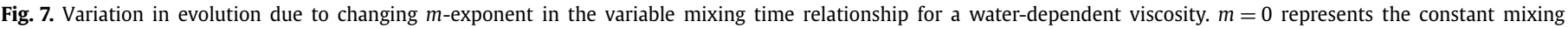

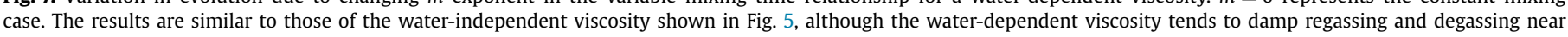
the start of the model. Deviation in (e) local water content is a result of increasing (f) mixing time, effectively trapping water in the mantle.

as evident. Mixing becomes more dependent on mantle conditions as $m$ increases and as result, net degassing occurs earlier and lasts for a shorter period of time.

By varying both $\tau$ and $m$, behaviour in the first $\sim 4$ Gyrs is dominated by evolution in the first few hundred million years. This implies that the history of the mantle water content and surface ocean is very sensitive to the initial mixing conditions. As such, we also test cases where the starting condition has a wet mantle and a dry surface (Figs. A1 and A2). The evolution is characterised by a rapid period of net degassing followed by the same behaviour as described above. This suggests that regardless of the starting conditions, net degassing may occur.

The net degassing period will also depend on the efficiency of the degassing process. $F_{d}=1$ in all of these models, assuming the most efficient transport to the surface. Whilst lower values are unexpected (Rüpke et al., 2013), values of $F_{d}$ ranging from 0 to 1 are tested (Fig. A4) show that the amplitude of net degassing is most affected. $F_{d}<1$ decreases how much water is degassed, though there is little change in the start and end times of the net degassing period. Further decreasing $F_{d}<0.4$ stops net degassing from occurring where $F_{d}=0$ results in an evolution where only regassing operates.

\subsection{Surface ocean volume}

Fig. 9b shows the surface ocean volume at 4.6 Gyrs of each case examined in Fig. 8. When the surface ocean is zero, all the water has been subducted into the mantle (Table 1). The majority of cases show less water at the surface at 4.6 Gyrs than for the instantaneous mixing case, i.e. where $\tau=0$; as the mixing time increases, the surface ocean volume decreases. Deviations from these features are between 0.5 and 4 Gyrs. These cases finish during or soon after the end of a period of net degassing. As net degassing lowers average mantle water content $X$, more water is found in the surface reservoir. These cases can end with a larger surface ocean than the instantaneous case. Even if these cases have gone through the end of net regassing, it still takes time for water content to recover and to be returned to the mantle. Therefore, cases that do not finish in a state of net degassing can still feel the effects of this period.

Constant mixing cases (orange in Fig. 9b) can show higher surface ocean volumes in comparison to the instantaneous case. Periods of net degassing are longer allowing more water to be released to the surface reservoir, resulting in less water in the mantle. When mixing is variable and the viscosity law is also waterdependent the surface ocean volume decreases and the effect of net degassing in the few cases where it does occur, is negligible. It is clear from Fig. 9b, that when mixing is included, variations in the surface ocean mass and hence the average mantle, can be up to an ocean. In general, as the mixing time is increased, the size of the surface ocean diminishes, unless the model is in a state of net degassing or is still recovering from one.

\subsection{Implications for Earth}

Thus far, this study has shown that Earth may go through more than one transition in the water cycle. It is clear that the incorporation of mixing time into the parametrised model has a large effect on the water content and can lead to extended periods of net degassing. Constant mixing 'locks' water in the mantle for a finite period of time. However, for variable mixing, cooling acts to progressively increase the mixing time, effectively trapping water 
(a) Constant Mixing \& $\eta(T)$

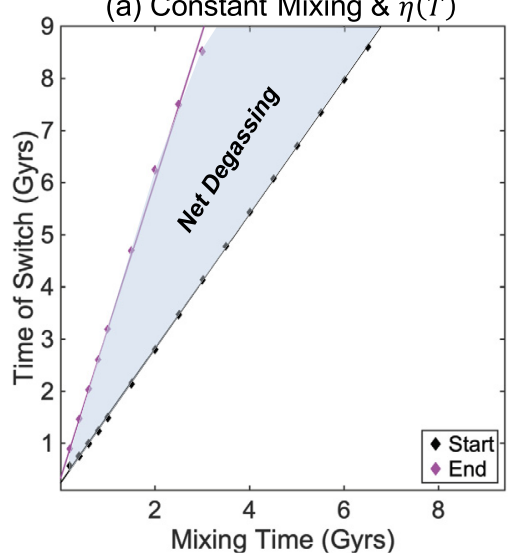

(b) Constant Mixing \& $\eta(T, X)$

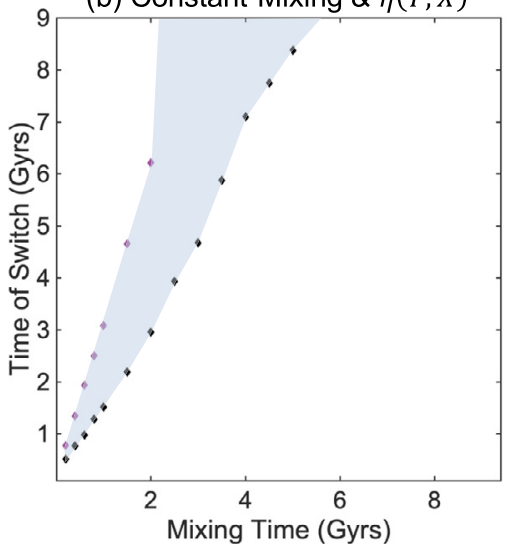

(c) Variable Mixing \& $\eta(T)$

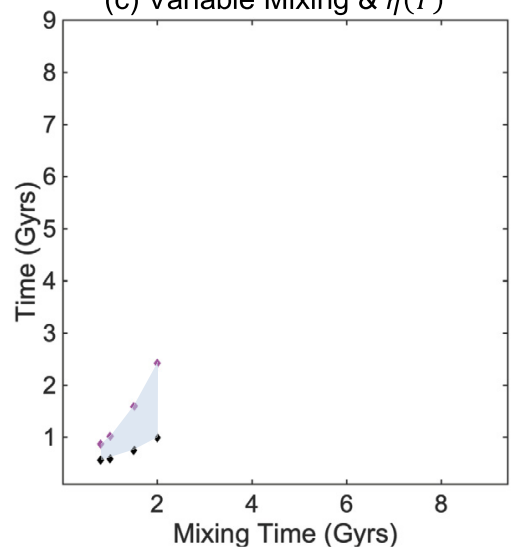

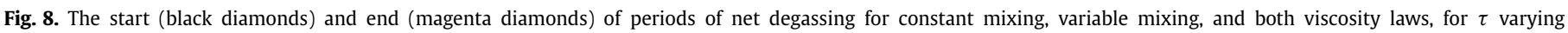

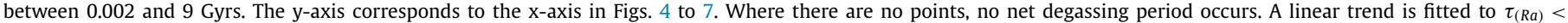

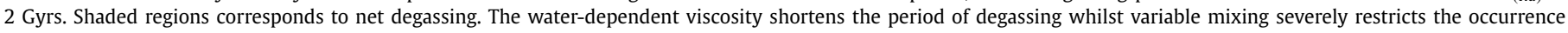
of degassing. No net degassing period occurs for variable mixing and water-dependent viscosity.

(a)

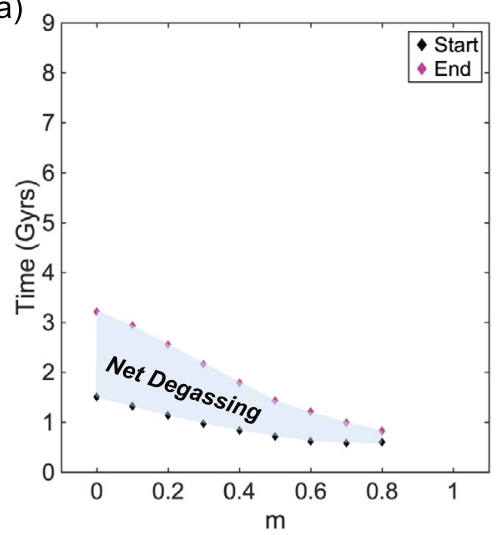

(b)

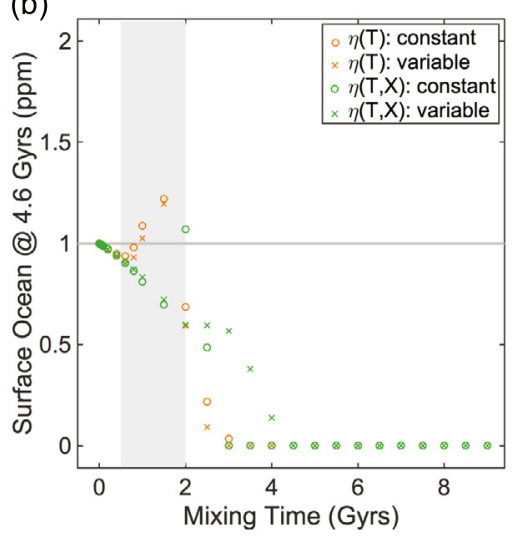

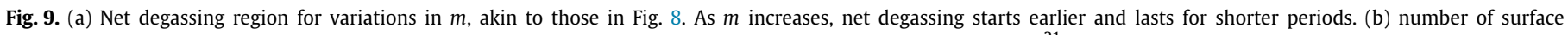

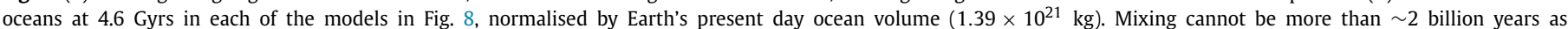

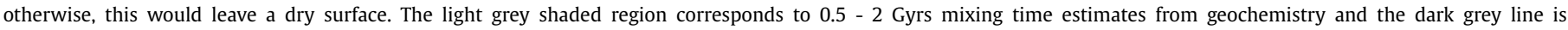

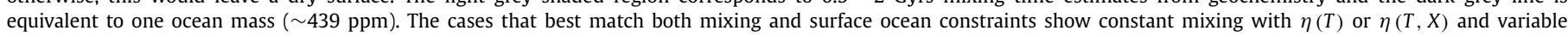
mixing with $\eta(T)$ are equally likely.

in the mantle. As the mixing time increases, mixing becomes more inefficient and it takes longer for water to reach a MOR. Less water is available to be degassed, trapping water at depth and keeping the mantle in a state of net regassing.

The cases presented are in agreement with others (e.g. Korenaga et al., 2017; Nakagawa and Spiegelman, 2017) where net regassing dominates. However, unlike previous parametrised models, we start with a large surface ocean rather than a hydrous mantle (Crowley et al., 2011; Sandu et al., 2011). This results in the loss of an initial degassing phase that stiffens the mantle and induces heating (also present when models start with wet mantle), although heating is still induced by the lack of water in the mantle at the beginning of the model. Cases with variable mixing are also in agreement with previous models where the mantle is not only hotter, but drier in the past, particularly during 0.5 and 2 Gyrs, corresponding to the Archean for Earth. As the planet has cooled, mixing time increases with decreasing $R a$ and the propensity to degas the mantle reservoir diminishes. When comparing Fig. 9b with current estimates for mixing times, 0.5-2 Gyrs (Gonnermann and Mukhopadhyay, 2009), and the present day ocean, only a few cases lie close to this range. Of these cases, none have a water-dependent viscosity and variable mixing time. The three closest points to one ocean mass and within $\tau=0.5-2$ Gyrs are $\tau=0.8$ Gyrs where mixing is constant and $\eta(T), \tau=1$ Gyrs where mixing is variable and $\eta(T)$ and $\tau=2$ Gyrs where mixing is constant and $\eta(T, X)$. However of these combinations, it is unclear which is most representative of Earth. It is clear that the mixing time cannot be more than $\sim 2$ billion years as otherwise water would be perpetually trapped in the mantle, never released to the surface.

Mixing has the greatest effect on temperature and water evolution when viscosity is independent of water content. Even if viscosity is not dependent on water (Muir and Brodholt, 2018), the water content in the melting region has the largest effect as $D$ is directly affected by $X$, pushing the system into a state of net degassing. Present day mixing times from geochemistry vary from $\sim 0.5-2$ Gyrs and up to $\sim 1$ Gyrs from geodynamic models (Tackley, 2015). But how this mixing time might have changed over the course of Earth evolution is unclear. For early Earth, Hadean mixing times on the scale of 0.1 Gyrs are predicted from geodynamic models with a mobile lid i.e. plate tectonics (e.g. Samuel et al., 2011; Kellogg and Stewart, 1991) whereas mixing in a stagnant lid model by O'Neill et al. (2013) suggests timescales more comparable to those presented by geochemistry, approximately 1 Gyrs, when tectonic regime is take into account. Constant mixing (Fig. 9b) shows two cases that potentially match one surface ocean at 4.6 Gyrs. Whilst there is uncertainty in mixing style (constant or variable) and the sensitivity of the mantle viscosity to water content (negligible or up to three orders of magnitude), it is clear that changes 
in the amount of water in the melt region at MORs can cause significant changes in the water cycle. This suggests that this effect of water content in the MOR region coupled with mixing may be more important than its relationship with viscosity when considering whole mantle evolution.

It is also interesting to consider the effect of a constant or variable mixing time on the overall behaviour of Earth as a dynamic system. For the case of instantaneous mixing (Fig. 6a), the Earth appears to behave similar to a simple first order system, where the mantle temperature declines continuously, with the rate of decay decreasing with time. The introduction of a constant mixing time leads to fluctuations in the temperature signal (Fig. 6a), similar to those seen in the early experimental study of time-delay systems by Callender et al. (1936). These fluctuations are less apparent when the mixing time is allowed to vary with time (Fig. 7a), especially for $m \gtrsim 0.2$; the apparent stabilisation of the system (and the resulting suppression of the period of net degassing) arises due to the reduction in the magnitude of the mixing time for large $m$ (Fig. 7f). This observation is consistent with previous studies of time-delay systems, for which complex dynamics and chaos occur when the time-delay is of a similar order to the characteristic response time of the system (Junges and Gallas, 2012). For much of the Earth's history, radiogenic heating has been responsible for the majority of heat flow through the mantle, and thus the time-scale of long term thermal evolution of the mantle is given by the relevant half lives, i.e. 1-10 Gyrs (Table A1). By the time the mantle has cooled sufficiently for mixing time to reach values comparable to the radiogenic time-scales (i.e. $\tau_{R a}=1 \mathrm{Gyrs}$, when chaotic behaviour may be expected), fluctuations in the temperature and water content are likely to have died out and the mantle is characterised by gradually decreasing temperature and increasing water content. In such a quasi-steady state, the mixing time will have a negligible effect in the long term. However, this may not be the case in the first few billion years, where the time-scales of mixing will be more comparable to the time-scales on thermal evolution.

These changes induced by the lag between regassing during subduction and degassing at MORs also has an effect on the surface reservoir (Fig. 9b). On Earth, as little as $\pm 1 \mathrm{ppm}$ can cause a change in today's global-mean sea level of $\pm 10 \mathrm{~m}$, indicating a significant contribution of mantle water to changes in sea level even if changes in the surface reservoir are on the order of a few ppm. Fig. 9b shows that these changes occur over hundreds of millions or billions of years. Today, global-mean sea level is rising at a rate of $\sim 3 \mathrm{~mm} / \mathrm{yr}$ (Watson et al., 2015) whereas changes in the constant mixing case (Fig. 4) are of the order $10^{-3} \mathrm{~mm} / \mathrm{yr}$. On short timescales, the mantle water contribution may not have much of an impact compared to ice sheet formation and other factors but over the evolution of the Earth would cause significant changes in the surface ocean volume and water content of the mantle. Even with lower estimates of mixing time ( $<1 \mathrm{Gyrs}$ ), changes in surface ocean mass can be up to 0.1 ocean masses, which would change sea level by $\sim 400 \mathrm{~m}$. During the initial phase of net regassing, the surface ocean diminishes and loses $\sim 1$ ocean to the mantle leaving less than an ocean at the surface, which could easily uncover highs in the sea floor. For the Earth, we can consider the onset of plate tectonics $\sim 2.5 \mathrm{Ga}$ or earlier. If we assume that this is also the beginning of the relatively efficient transport of water into the deep mantle, topography could easily become uncovered and enhance subaerial weathering, an important process aiding the formation of continental crust (Höning et al., 2014). Sea level is the ideal observable to consider with further constraints on the surface ocean from geological data (e.g. Korenaga et al., 2017). Tracking the sea level on the time scale since the break up of Pangea has shown a decrease in ocean mass (Karlsen et al., 2019). Further models in times of sustained super continents with plate reconstructions could show delays between period of rapid subduction and super- continent break-up in response to melting of relatively hydrated material. Understanding transitions on the billion year time scale requires a different set of tools. Recent work on atmospheric xenon isotopes has shown that there has been at least one transition from net degassing to regassing (Parai and Mukhopadhyay, 2018) on the billion year timescale and further work with this outlook could illuminate more times in Earth's history when similar transitions may occur. This would also require understanding how water is transported in different tectonic regimes (e.g. Sleep et al., 2014) such as heat pipe (Moore and Webb, 2013), 'squishy lid' tectonics (Lourenço et al., 2018) and incorporation into complex, 2D and 3D models. Hints of net degassing have been seen in 2D (Nakagawa and Nakakuki, 2019) and 3D (Price et al., 2019) models, but not yet discussed. Nakagawa and Nakakuki (2019) show transitions on 0.1 Gyrs scale whereas Price et al. (2019) show cases where net degassing occurs for 0.5 Gyrs. Variations are on the scale of $130 \mathrm{ppm}$ which would correspond to changes in over $1 \mathrm{~km}$ of sea level. Further dimensional models investigating these changes are required to aid the understanding of mixing and it's impact on the surface ocean.

\section{Conclusions}

We present a parametrised model (Crowley et al., 2011) adapted to include the coupled effects of mixing and a waterdependent viscosity to explore the effects of mixing on mantle water content and surface ocean volume. The introduction of a second water content $X\left[\tau_{(R a)}\right]$ results in degassing controlled by a local water content, different from the average mantle water content. This results in a period of net degassing where mantle water content decreases and the surface ocean volume increases. The impact of changing water content in the melt region facilitates the appearance of a net degassing period, having a larger effect on the overall water cycle evolution than a water-dependent viscosity. The mixing time itself cannot be more than $\sim 2$ billion years as values greater than this would result in a dry surface. Lastly, even small variations in ocean mass can cause large variations in sea level and once subduction became a viable water transport mechanism, it may have aided sub-aerial weathering in the Early Earth.

\section{Acknowledgements}

Firstly, thank you to John Crowley who generously shared his 2011 code along with some sage advice. Thanks also to Takashi Nakagawa and William B. Moore whose comments helped to greatly improve this manuscript. This work was funded by Natural Environment Research Council (NERC) as part of the Deep Volatiles Consortium (NE/M00046X/1) and the Louis B and Martha B Slichter Endowed Funds.

\section{Appendix A. Supplementary material}

Supplementary material related to this article can be found online at https://doi.org/10.1016/j.epsl.2019.115881.

\section{References}

Ammann, M., Brodholt, J., Dobson, D., 2010. Simulating diffusion. Rev. Mineral. Geochem. 71 (1), 201-224.

Callender, A., Hartree, D.R., Porter, A., 1936. Time-lag in a control system philosophical. Philos. Trans. R. Soc., Math. Phys. Eng. Sci. 756, 415-444. https:// doi.org/10.1098/rsta.1936.0007.

Crowley, J.W., Gérault, M., O'Connell, R.J., 2011. On the relative influence of heat and water transport on planetary dynamics. Earth Planet. Sci. Lett. 310 (3-4) 380-388.

Doyne Farmer, J., 1982. Chaotic attractors of an infinite-dimensional dynamical system. Phys. D, Nonlinear Phenom. 4 (3), 366-393. 
Elkins-Tanton, L.T., 2011. Formation of early water oceans on rocky planets. Astrophys. Space Sci. 332 (2), 359-364.

Fei, H., Wiedenbeck, M., Yamazaki, D., Katsura, T., 2013. Small effect of water on upper-mantle rheology based on silicon self-diffusion coefficients. Nature 498 (7453), 213-215.

Gonnermann, H.M., Mukhopadhyay, S., 2009. Preserving noble gases in a convecting mantle. Nature 459 (7246), 560-563.

Hirschmann, M.M., Tenner, T., Aubaud, C., Withers, A.C., 2009. Dehydration melting of nominally anhydrous mantle: the primacy of partitioning. Phys. Earth Planet. Inter. $176(1-2), 54-68$

Höning, D., Hansen-Goos, H., Airo, A., Spohn, T., 2014. Biotic vs. abiotic Earth: a model for mantle hydration and continental coverage. Planet. Space Sci. 98, $5-13$.

Höning, D., Spohn, T., 2016. Continental growth and mantle hydration as intertwined feedback cycles in the thermal evolution of Earth. Phys. Earth Planet. Inter. 255, 27-49.

Junges, L., Gallas, J.A., 2012. Intricate routes to chaos in the Mackey-Glass delayed feedback system. Phys. Lett. A 376 (30-31), 2109-2116.

Karlsen, K.S., Conrad, C.P., Magni, V., 2019. Deep water cycling and sea level change since the breakup of Pangea. Geochem. Geophys. Geosyst. 20 (6). https://doi.org/ 10.1029/2019GC008232.

Kellogg, L.H., Stewart, C.A., 1991. Mixing by chaotic convection in an infinite Prandt number fluid and implications for mantle convection. Phys. Fluids A, Fluid Dyn. 3 (5), 1374.

Korenaga, J., 2011. Thermal evolution with a hydrating mantle and the initiation of plate tectonics in the early Earth. J. Geophys. Res. 116 (B12), B12403.

Korenaga, J., Planavsky, N.J., Evans, D.A.D., 2017. Global water cycle and the coevolution of the Earth's interior and surface environment. Philos. Trans. R. Soc., Math. Phys. Eng. Sci. 375 (2094), 20150393.

Lay, T., Buffett, B., 2008. CMB-heat flow. Nat. Geosci., 13-15.

Lourenço, D.L., Rozel, A.B., Gerya, T., Tackley, P.J., 2018. Efficient cooling of rocky planets by intrusive magmatism. Nat. Geosci. 11 (5), 322-327.

Mackey, M., Glass, L., 1977. Oscillation and chaos in physiological control systems. Science 197 (4300), 287-289.

Marty, B., Avice, G., Sano, Y., Altwegg, K., Balsiger, H., Hässig, M., Morbidelli, A., Mousis, O., Rubin, M., 2016. Origins of volatile elements (H, C, N, noble gases) on Earth and Mars in light of recent results from the ROSETTA cometary mission. Earth Planet. Sci. Lett. 441, 91-102.

May, R.M., 1980. Non-linear phenomena in ecology and epidemiology. Ann. N.Y. Acad. Sci. 357 (1), 267-281.

Mckenzie, D., Bickle, M.J., 1988. The volume and composition of melt generated by extension of the lithosphere. J. Petrol. 29 (3), 625-679.

Mei, S., Kohlstedt, D.L., 2000. Influence of water on plastic deformation of olivine aggregates: 2. Dislocation creep regime. J. Geophys. Res. 105 (B9), 21471.

Moore, W.B., Webb, A.A.G., 2013. Heat-pipe Earth. Nature 501 (7468), 501-505.

Muir, J.M., Brodholt, J.P., 2018. Water distribution in the lower mantle: implications for hydrolytic weakening. Earth Planet. Sci. Lett. 484, 363-369.
Nakagawa, T., Nakakuki, T., 2019. Dynamics in the uppermost lower mantle: insights into the deep mantle water cycle based on the numerical modeling of subducted slabs and global-scale mantle dynamics. Annu. Rev. Earth Planet. Sci. 47 (1), 41-66.

Nakagawa, T., Nakakuki, T., Iwamori, H., 2015. Water circulation and global mantle dynamics: insight from numerical modeling. Geochem. Geophys. Geosyst. 16 (5), 1449-1464.

Nakagawa, T., Spiegelman, M.W., 2017. Global-scale water circulation in the Earth's mantle: implications for the mantle water budget in the early Earth. Earth Planet. Sci. Lett. 464, 189-199.

Niculescu, S.-I., Verriest, E.I., Dugard, L., Dion, J.-M., 1998. Stability and robust stability of time-delay systems: a guided tour. In: Stability and Control of Time-Delay Systems. Springer-Verlag, London, pp. 1-71.

Ohtani, E., Mizobata, H., Yurimoto, H., 2000. Stability of dense hydrous magnesium silicate phases in the systems Mg2SiO4-H2O and MgSiO3-H2O at pressures up to 27 GPa. Phys. Chem. Miner. 27 (8), 533-544.

O'Neill, C., Debaille, V., Griffin, W., 2013. Deep Earth recycling in the Hadean and constraints on surface tectonics. Am. J. Sci. 313 (9), 912-932.

Parai, R., Mukhopadhyay, S., 2018. Xenon isotopic constraints on the history of volatile recycling into the mantle. Nature 560 (7717), 223-227.

Price, M.G., Davies, J.H., Panton, J., 2019. Controls on the deep-water cycle within three-dimensional mantle convection models. Geochem. Geophys. Geosyst. 20 (5). https://doi.org/10.1029/2018GC008158.

Rüpke, L., Phipps Morgan, J., Eaby Dixon, J., 2013. Implications of subduction rehydration for Earth's deep water cycle. EOS Trans. AGU 87, 263-276.

Samuel, H., Aleksandrov, V., Deo, B., 2011. The effect of continents on mantle convective stirring. Geophys. Res. Lett. 38 (4), 1-5.

Sandu, C., Lenardic, A., McGovern, P., 2011. The effects of deep water cycling on planetary thermal evolution. J. Geophys. Res., Solid Earth 116 (12), 1-16.

Sleep, N.H., Zahnle, K.J., Lupu, R.E., 2014. Terrestrial aftermath of the Moon-forming impact. Philos. Trans. R. Soc., Math. Phys. Eng. Sci. 372 (2024).

Tackley, P., 2015. Mantle geochemical geodynamics. In: Treatise on Geophysics, vol. 7. Elsevier, pp. 521-585.

Tikoo, S.M., Elkins-Tanton, L.T., 2017. The fate of water within Earth and superEarths and implications for plate tectonics. Philos. Trans. R. Soc., Math. Phys. Eng. Sci. 375 (2094), 20150394.

Tucker, J.M., Mukhopadhyay, S., 2014. Evidence for multiple magma ocean outgassing and atmospheric loss episodes from mantle noble gases. Earth Planet. Sci. Lett. 393, 254-265.

Turcotte, D.L., Oxburgh, E.R., 1967. Finite amplitude convective cells and continental drift. J. Fluid Mech. 28 (01), 29-42.

Watson, C.S., White, N.J., Church, J.A., King, M.A., Burgette, R.J., Legresy, B., 2015. Unabated global mean sea-level rise over the satellite altimeter era. Nature Clim. Change 5 (6), 565-568. 